\title{
Diabetic Ketoacidosis: Demographic Data, Clinical Profile and Outcome in a Tertiary Care Hospital
}

\author{
Zahra Razavi, ${ }^{1,}{ }^{*}$ and Farimehr Hamidi ${ }^{1}$ \\ ${ }^{1}$ Department of Pediatrics, Hamedan University of Medical Sciences, Hamedan, Iran \\ "Corresponding author: Zahra Razavi, Pediatrics Department, Besat Hospital, Motahari Boulevard, Resalat Square, Hamedan, Iran. Tel: +98-9183122066, E-mail: \\ razavizahra@yahoo.com.au
}

Received 2016 June 23; Revised 2017 February 12; Accepted 2017 March 19.

\begin{abstract}
Background: Diabetic ketoacidosis (DKA) is an acute and life-threatening situation that accounts for the majority of diabetesrelated morbidity and mortality in children and adolescents who suffer from type 1 diabetes mellitus (T1DM).

Objectives: To investigate the demographic, clinical characteristics, and outcomes of DKA in young patients with established T1DM and newly diagnosed diabetes in a tertiary referral hospital.

Methods: Data from all T1DM patients diagnosed with DKA episodes in the pediatric endocrine unit at Besat university hospital, Hameden, Iran during 2006-2013 were reviewed in a retrospective study. The data collected includes the demographic data (age, sex, place of residence, first presentation and established T1DM), clinical presentation (main presenting symptoms, average duration of presenting symptoms before hospitalization, precipitating factors, severity of DKA, level of consciousness), laboratory parameters (blood sugar, arterial blood gases, urine ketones, serum electrolytes) and the outcome. DKA was defined as a glucose level higher than $250 \mathrm{mg} / \mathrm{dL}, \mathrm{pH}<7.30$, bicarbonate $>15 \mathrm{mmol} / \mathrm{L}$ and ketonuria. Data was entered and analyzed on SPSS version 16 . A P value less than 0.05 were defined as statistically significant.

Results: The study population comprised 72 children satisfying the inclusion criteria of the study. Mean age of patients was 9.4 \pm 4.08 years (range 5 months-18.2 years). The majority (61.1\%) of patients were urban residents. DKA occurrence was higher (29.2\%) in summer. Newly diagnosed diabetics accounted for $59(81.9 \%)$ of the patients. The majority of the patients $(54.2 \%)$ were aged 10 - 14 years. The median duration of symptoms before the hospitalization was $9.5 \pm 9$ days. The most commonly reported presenting symptoms were polyuria (86.1\%), polydipsia (84.7\%) followed by gastrointestinal symptoms. Nearly $40.2 \%$ of patients presented with an altered level of consciousness. About half of patients had a medical encounter before diagnosis. The mean initial blood glucose was $423 \pm 96 \mathrm{mg} / \mathrm{dL}$. Severe DKA was occurring more frequently than moderate and mild forms ( $47.2 \%$, versus $34.7 \%$ and $18.1 \%$, respectively). DKA was significantly more severe in girls $(\mathrm{P}=0.004)$. Average time of recovery was 21 hours $($ range $4-75 \mathrm{~h})$. The commonest complication was hypokalemia (34.7\%) followed by hypernatremia (26.4\%). There was a significant relationship between hypokalemia and clinical severity $(\mathrm{P}=0.02)$, and between abdominal pain and severity of DKA $(\mathrm{P}=0.003)$. No deaths occurred.

Conclusions: DKA was most prevalent in newly diagnosed TIDM cases. Most cases had severe DKA. Female children, in particular, seem to be at increased risk for severe DKA. Newly diagnosed T1DM and insulin omission were the main factors associated with DKA. The age of presentation and clinical symptoms of studied subjects were similar to international studies. No lethal complication was recorded. High frequency of DKA at presentation of T1DM requires careful attention to issues of early diagnosis before development of ketoacidosis.
\end{abstract}

Keywords: Hyperglycemia, Diabetes Mellitus Type 1, Ketoacidosis, Children

\section{Background}

Ketoacidosis is a serious complication of T1DM and the leading cause of diabetes-related deaths, mainly due to cerebral edema that occurs in $0.4 \%-3.1 \%$ of patients (1-3). It also accounts for a large proportion of diabetes-related hospitalizations $(4,5)$. The risk of DKA in young patients with established TIDM is $1 \%-10 \%$ per patient per year (6). International studies indicate that $15 \%-70 \%$ of patients with newly onset T1DM present in diabetic ketoacidosis (7, 8). Insulin deficiency makes children with diabetes at onset vulnerable to DKA. Likewise, a failure in insulin admin- stration, acute stress, lack of insulin therapy during intercurrent illness, poor metabolic control, inappropriate interruption of insulin pump therapy, psychiatric disorders, difficult family circumstances, lower socioeconomic status, and lack of appropriate health insurance can develop DKA in patients suffering from established diabetes $(6,9)$. DKA most often can be found in patients diagnosed with T1DM, however, patients with type 2 diabetes are also susceptible to DKA under stress-inducing situations, such as trauma, surgery, or infections $(8,10)$.

Considering the worldwide variation in demographic characteristics and outcome of DKA, regional studies on 
this subject seem to be rational. DKA rates can be reduced or prevented by identifying and targeting associated risk factors.

\section{Objectives}

The purpose of this study was to analyze the clinical profile, laboratory data, and outcome of children and adolescents with DKA during a period of 7 years (2006 - 2013) in the Besat university hospital in Hamedan. The results of this survey would contribute to design intervention strategies to decrease the rate of DKA.

\section{Methods}

The study was a retrospective case record review. All medical files of children with discharge diagnosis of DKA were enrolled. We analyzed data related to demographics, clinical presentation, biochemical, management and outcome of all the cases.

\subsection{Participants, Data Acquisition, and Variable Definition}

Eligible subjects were aged $<19$ years. All patients who received intravenous rehydration and insulin infusion prior to admission in our center and those without the required information of the study were excluded. DKA was diagnosed if blood glucose $>250 \mathrm{mg} / \mathrm{dL}$, venous $\mathrm{pH}<7.3$ or bicarbonate $<15 \mathrm{mmol} / \mathrm{L}$ and ketonuria were detected (8).

ISPAD 2014 guidelines were used to define DKA severity categories. Accordingly, three groups were defined; (1) mild (venous $\mathrm{pH}<7.3$ or bicarbonate $<15 \mathrm{mmol} / \mathrm{l}$ ), (2) moderate ( $\mathrm{pH}<7.2$ or bicarbonate $<10 \mathrm{mmol} / \mathrm{l}$ ), and (3) severe ( $\mathrm{pH}<7.1$ or bicarbonate $<5 \mathrm{mmol} / \mathrm{l})(11,12)$. Hypoglycemia was defined as blood sugar $<60 \mathrm{mg} / \mathrm{dL}$, hypokalemia: serum potassium $<3 \mathrm{mEq} / \mathrm{L}$, hyperkalemia: serum potassium $>6 \mathrm{mEq} / \mathrm{L}$, hyponatremia: corrected serum sodium $<135 \mathrm{mEq} / \mathrm{L}$, hypernatremia: serum sodium $>160 \mathrm{mEq} / \mathrm{L}$ (8). Time for resolution of DKA was extracted from patients' medical records. The criteria for DKA resolution were as follows: general condition improvement, full consciousness, normal vital signs, oral feeding tolerance without emesis and resolution of acidosis (Normal pH and serum bicarbonate of $>18 \mathrm{mEq} / \mathrm{L}$ was considered as resolution of acidosis). Outcome of DKA was defined in terms of death or survival without considering any acute or chronic problems during the one year follow-up, e.g. cerebral edema and related consequences, renal failure, rhabdomyolysis, stroke, deep vein thrombosis (DVT), pneumomediastinum, acute respiratory distress syndrome (ARDS) with pulmonary edema, and gastrointestinal complications (1113).

\subsection{Ethical Issue}

The present study was approved by the local ethics committee and research of Hamedan University of Medical Sciences (Study number: p/16/70/2/1813).

\subsection{Statistical Analysis}

Statistical analysis was performed using SPSS ver.16.0. The qualitative data of patients were analyzed using chi square and (expressed as percentages). The independent two-sample t-test was used to compare the quantitative variables and to analyze the differences between means. Some data including sex ratio, difference between the DKA frequency, and season were compared using chi-square test. One way ANOVA was used to determine the association between age groups and the severity of ketoacidosis. The data were presented as means \pm standard deviations. Statistical significance was defined as $\mathrm{P}<0.05$ for all clinical and laboratory data.

\section{Results}

Thirteen medical records had incomplete data and, consequently, were excluded from the study. Overall, 72 patients ( $44=61.1 \%$ female, $28=39.9 \%$ male) with ages less than 19 years fulfilled all the inclusion criteria. Desired characteristics of included cases are summarized in Table 1. Mean age of patients was $9.4 \pm 4.08$ years (range between 5 months and 18.2 years). The majority of patients were urban (61.1\% versus $38.9 \%$ ) residents. DKA occurrence was higher (29.2\%) in summer. Hospitalization due to ketoacidosis was higher in December (13.9\%) and September (11.12\%) than in other months. No significant seasonality was observed $\left(X^{2}=8,00, P=0.7\right)$. The majority of patients (59 cases, 81.9\%) were newly diagnosed T1DM and 13 (18.1 $\%$ ) cases had established T1DM. The majority (54.2\%) of the patients were in the age group 10-14 yrs. The median duration of symptoms before referring to hospital was $9 \pm$ 9.5 days. The most commonly reported presenting symptoms were polyuria (86\%), polydipsia (84.7\%) followed by gastrointestinal symptoms, including nausea or vomiting (47.2\%) and abdominal pain (43.1\%). A total of $40.2 \%$ of the patients presented with an altered level of consciousness and $6.9 \%$ of them were in deep coma. About half of the patients (37 cases, 51.1\%) had not been visited by local physician prior to admission with DKA, whereas nearly $48.6 \%$ of them were seen by physicians at least one time prior to the DKA diagnosis, but physicians failed to immediately make the diagnosis of IDDM. Out of the 13 subjects with established diabetes, the promoting factor for DKA was insulin omission in 7 (9.7\%) children, whereas in 6 children the 
cause was unknown. Details of laboratory profiles and the outcome of the studied patients are shown in Table 2.

The mean initial blood glucose was $423 \pm 96 \mathrm{mg} / \mathrm{dL}$. Severe DKA had occurred more frequently than moderate and mild forms (47.2\% vs $34.7 \%$ and $18.1 \%$, respectively). DKA was significantly more severe in females than in males $(\mathrm{P}=$ 0.004 ). None of the patients received intravenous sodium bicarbonate. Average time of recovery from DKA was 21 (4 - 75) hours. There was no significant relationship between age or living location and clinical severity $(\mathrm{P}=0.7, \mathrm{P}=0.2$, respectively), and between known or new diabetics and severity of DKA $(\mathrm{P}=0.5)$. The commonest complication was hypokalemia (34.7\%) followed by hyponatraemia (26.4\%). There was a significant relationship between hypokalemia and clinical severity $(\mathrm{P}=0.02)$, and between abdominal pain and severity of DKA $(\mathrm{P}=0.003)$. All cases received appropriate treatment and recovered without lethal complications. No deaths occurred. All the patients collaborated well during the 12 months follow-up.

\section{Discussion}

This retrospective study described the demographic data and clinical profile of children with DKA in a tertiary care hospital. DKA, as the initial presentation, was encountered by the vast majority of patients. Classical symptoms of DKA namely polyuria, polydipsia, abdominal pain, vomiting, and decreased level of consciousness were the presenting complaints in most cases. New onset diabetes and insulin omission in those with established T1DM were the most common precipitants of DKA. Seasonal pattern was not evident at diagnosis. Approximately, half of patients (54.2\%) were above 10 years old. This finding may be explained by the fact that a high number of patients were new cases of T1DM. As we know, the second and much greater peak incidence of diabetes in children is during early puberty (i.e. ages between 10 - 14 years) (14). These data, therefore, were attributed to the high number of new-onset cases presenting with DKA. The age of presentation in our children is similar to that reported in international studies $(8,15,16)$. Lone et al. (8) reported that $55 \%$ of children presenting with DKA were above 10 years old. Naeem et al. (16) by examining 373 pediatric DKA patients reported that number of attacks were highest in patients who aged 10-14 years. This finding is contrary to the results of some previous authors who demonstrated that diabetic ketoacidosis was more common in younger ages (17-20).

This study showed that the frequency of hospitalizations for DKA was higher in summer and autumn and lower in spring. However, differences were not significant. We have previously shown that the frequency of T1DM was higher in summer (34\%) (21). Since the majority of DKA cases occur in patients without a known history of diabetes, its seasonality is also a measure of new onset T1DM seasonality. Thus, we conclude that seasonal variation of DKA and T1DM in our region is slightly different from those reported in the literature, while confirming our previous findings (22).

Similar to the results of Butalia et al. (23), hospitalization due to ketoacidosis was higher in December and September than in other months. So, healthcare professionals' alertness may need to be increased, particularly during certain months of the year. Polyuria, polydipsia, abdominal pain, vomiting, and altered level of consciousness were the commonest presenting complaints of DKA consistent with findings in previous studies $(16,24)$.

In line with our previous study in 2010 (25), severe DKA was observed in $47.2 \%$ of patients which differs from other studies as they found a lower number of severe DKA compared with mild and moderate type. For instance, Schober et al. (26) reported that of 1,238 Austrian children with DKA, 383 (11.5\%) had severe DKA. In the study of Oyarzabal et al. (15) severe DKA was diagnosed in $17.8 \%$ DKA episodes. The DKA episodes were severe in $49.4 \%$, consistent with the results of Guisado-Vasco et al'. study (27). The possible reason may be due to a lack of access to healthcare professionals for the study population or delayed diagnosis by primary care physician. Recognition of DKA can be improved by increasing health care provision and facilities as well as healthcare professionals' alertness.

In this work, DKA was found significantly more severe in female children. The reason for this finding cannot be explained. Our data show no association between age and severity of DKA. The data confirm the results of other studies indicating that age was not significantly associated with the severity of DKA (28-30). In contrast Jesic et al. (31) reported that severe DKA was more frequent in the age group $<5$ years.

Our data also indicated no association between newly diagnosed diabetes and severity of DKA. The mean blood glucose level observed at the time of DKA presentation was close to what was stated by other studies (32).

Regarding the number of visits preceding diagnosis, nearly $48.6 \%$ of the study group were seen by physicians at least once prior to DKA diagnosis. Similarly, Bui et al. (33) realized that $38.8 \%$ of 285 children with DKA had at least one medical visit before admission. Given that DKA is preceded by a variable course of symptomatic diabetes characterized by polyuria and polydipsia, the challenge for future strategies should be finding effective ways to raise the awareness of healthcare professionals to early diagnosis of T1DM in children before the development of ketoacidosis. A high number of cases were at their first presentation of T1DM, implying that more attention and prevention strate- 
Table 1. Demographic Features and Characteristics of Studied Cases on Admission

\begin{tabular}{|c|c|c|}
\hline Characteristics & Categories & Results \\
\hline Mean age at diagnosis (year), mean (SD) & $\mathrm{N} / \mathrm{A}$ & $9.4 \pm 4.08$ years \\
\hline Median duration of symptoms (days), mean (SD) & $\mathrm{N} / \mathrm{A}$ & $9.5 \pm 9$ \\
\hline \multirow{2}{*}{ Gender, $\mathbf{n}(\%)$} & Female & $44(61.1)$ \\
\hline & Male & $28(38.9)$ \\
\hline \multirow{4}{*}{ Age distribution at diagnosis (year), $n$ (\%) } & $0-4$ & $10(13.9)$ \\
\hline & $5-9$ & $19(26.4)$ \\
\hline & $10-14$ & $39(54.2)$ \\
\hline & $15-19$ & $4(5.6)$ \\
\hline \multirow{4}{*}{ Season at presentation } & Spring & $13(18.1)$ \\
\hline & Summer & $21(29.2)$ \\
\hline & Autumn & $19(26.4)$ \\
\hline & Winter & $19(26.4)$ \\
\hline \multirow{2}{*}{ Living location, $n$ (\%) } & Urban & $44(61.1)$ \\
\hline & Rural & $28(38.9)$ \\
\hline \multirow{2}{*}{ Disease presentation } & Newly diagnosed & $59(81.9)$ \\
\hline & Established diabetes & $13(18.1)$ \\
\hline \multirow{5}{*}{ Presenting symptoms } & Polyuria & $62(86.1)$ \\
\hline & Polydipsia & $61(84.7)$ \\
\hline & Vomiting, nausea & $34(47.2)$ \\
\hline & Abdominal pain & $31(43.1)$ \\
\hline & Unconscious & $29(40.2)$ \\
\hline \multirow{3}{*}{ Number of medical visits before admission } & Zero & $37(51.4)$ \\
\hline & One & $27(37.5)$ \\
\hline & Two & $8(11.1)$ \\
\hline
\end{tabular}

Table 2. Laboratory Profiles and Outcome Among the Studied Patients

\begin{tabular}{l|c|c}
\hline Characteristics & Categories & Data \\
\hline Blood glucose (mg/dL), mean (SD) & N/A & $423 \pm 96$ \\
\hline pH mean(SD) & N/A & $7.06 \pm 0.68$ \\
\hline $\mathbf{C O}_{\mathbf{2}}$ mean (SD) & N/A & $15.11 \pm 6.5$ \\
\hline HCO $^{3}$ mean (SD) & N/A & $6.43 \pm 1.2$ \\
\hline \multirow{2}{*}{ Severity } & Mild & $18.1 \%$ \\
\cline { 2 - 3 } & Moderate & $34.7 \%$ \\
\cline { 2 - 3 } & Severe & $47.2 \%$ \\
\hline \multirow{2}{*}{ Complications, $\mathbf{n}(\%)$} & Hypokalemia & $25(34.7)$ \\
\cline { 2 - 3 } & Hyponatremia & $19(26.4)$ \\
\hline Mean recovery time(hours) & N/A & $21(4-75)$ \\
\hline
\end{tabular}

gies are needed for early diagnosis of diabetes in new patients.

We found that the duration of symptoms prior to admission was lower than what we observed in our previous study ( $9 \pm 9.5$ days versus $14.84 \pm 8.19$ days) (25). It is unclear whether this reflects particularly the occurrence of aggressive forms of the diabetes or a consequence of early diagnosis due to improvements in diabetes diagnosis and referral to secondary care centers. Our finding differs from studies carried out by Lone et al. (8) and Habib (34) as they found a longer duration of symptoms before the presentation with ketoacidosis ( 21 and 15.8 days, respectively)

It is noteworthy that a significant number (43.1\%) of patients had abdominal pain. We observed that abdominal pain occurred more in cases with severe metabolic acidosis. The pathogenesis of the abdominal pain in ketoacidosis so far has not been rigorously defined and may be mul- 
tifactorial, involving metabolic and electrolytic origin, gastric distention or stretching of the liver capsule $(34,35)$.

In support of Umpierrez et al. (36), we believe that abdominal pain accompanied by severe DKA represents severe electrolyte imbalances and dehydration.

The commonest complications were hypokalemia and hyponatraemia. Similar observations have been reported in previous studies $(37,38)$. In current work, all cases recovered without lethal complications and no deaths occurred. Whereas, some authors believe that $10 \%-25 \%$ of survivors of DKA have significant residual morbidity (39). This may be attributed to the intensive, appropriate and careful treatment and physicians' experience in our pediatric critical care.

This study found notable findings, although our center is a tertiary medical center for treating children with diabetes, the frequency of hospitalizations for DKA is low. This is likely owned to the presence of experienced practitioners in diagnosis and treatment of DKA in the non-specialist settings.

Limitations: Our study has several limitations that warrant consideration. First, this is a retrospective single center study, consequently, caution should be exercised in generalizing the findings to all young patients with DKA in Iran. Second, the relatively small sample size limits the study's statistical power. Finally, we had to exclude thirteen medical records due to incomplete data which might have led to selection bias in our study. Despite these important limitations, our study gives a basic profile of DKA in our region.

\subsection{Conclusions}

Results of this study add to previous researches by providing evidence on characteristics and outcome of children with DKA. Overall, high number of patients was at the first time of presentation. Newly diagnosed T1DM and insulin omission were the main factors associated with DKA. Data concerning precipitating factor of DKA, age presentation, and clinical symptoms of the studied subjects were similar to international studies. Severe DKA was occurring more frequently than moderate and mild forms.

All patients had good prognosis and no deaths were reported. Awareness of diabetes symptoms, particularly in girls, needs more attention. High frequency of DKA at the presentation of T1DM requires careful attention to issues of early diagnosis before the development of ketoacidosis. Our study emphasizes that further efforts to reduce the occurrence of DKA must be focused upon earlier diagnosis of T1DM in order to decrease DKA at diagnosis.

\section{Acknowledgments}

This paper was extracted from a PhD thesis by Farimehr Hamidi. The authors would like to express their thanks to all medical records department staff at Besat hospital for their cooperation in data collecting. They wish to thank Dr. Mohammad Ali Sifrabiei for his statistical consulting. They also appreciate Mojgan Shahbazi member of the research center of Hamedan Besat Hospital for her skillful assistance in completion of this work.

\section{References}

1. Australian Paediatric Endocrine Group A. . Guidelines on the management of diabetes in children and adolescents. National Health Res Council Australia,. 2005.

2. Lawrence SE. Diagnosis and treatment of diabetic ketoacidosis in children and adolescents. Paediatr Child Health. 2005;10(1):21-4. [PubMed: 19657439].

3. Skitch SA, Valani R. Treatment of Pediatric Diabetic Ketoacidosis in Canada: A Review of Treatment Protocols from Canadian Pediatric Emergency Departments. CJEM. 2015;17(6):656-61. doi: 10.1017/cem.2015.56. [PubMed: 26461431].

4. Siafarikas A, O'Connell S. Type 1 diabetes in children - emergency management. Aust Fam Physician. 2010;39(5):290-3. [PubMed: 20485715].

5. Weintrob N, Phillip M. [Diabetic ketoacidosis in children and adolescents]. Harefuah. 2007;146(12):945-51. [PubMed: 18254447] 997-8.

6. Dunger DB, Sperling MA, Acerini CL, Bohn DJ, Daneman D, Danne TP, et al. European Society for Paediatric Endocrinology/Lawson Wilkins Pediatric Endocrine Society consensus statement on diabetic ketoacidosis in children and adolescents. Pediatrics. 2004;113(2):133-40. [PubMed: 14754983].

7. Lawrence SE, Cummings EA, Gaboury I, Daneman D. Populationbased study of incidence and risk factors for cerebral edema in pediatric diabetic ketoacidosis. J Pediatr. 2005;146(5):688-92. doi: 10.1016/j.jpeds.2004.12.041. [PubMed: 15870676].

8. Lone SW, Siddiqui EU, Muhammed F, Atta I, Ibrahim MN, Raza J. Frequency, clinical characteristics and outcome of diabetic ketoacidosis in children with type-1 diabetes at a tertiary care hospital. J Pak Med Assoc. 2010;60(9):725-9. [PubMed: 21381577].

9. Vanelli M, Chiarelli F. Treatment of diabetic ketoacidosis in children and adolescents. Acta Biomed. 2003;74(2):59-68. [PubMed: 14509913].

10. Fasanmade OA, Odeniyi IA, Ogbera AO. Diabetic ketoacidosis: diagnosis and management. Afr J Med Med Sci. 2008;37(2):99-105. [PubMed: 18939392].

11. Muir AB, Quisling RG, Yang MC, Rosenbloom AL. Cerebral edema in childhood diabetic ketoacidosis: natural history, radiographic findings, and early identification. Diabetes Care. 2004;27(7):1541-6. [PubMed: 15220225].

12. Ghetti S, Lee JK, Sims CE, Demaster DM, Glaser NS. Diabetic ketoacidosis and memory dysfunction in children with type 1 diabetes. $J P e$ diatr. 2010;156(1):109-14. doi: 10.1016/j.jpeds.2009.07.054. [PubMed: 19833353].

13. Bialo SR, Agrawal S, Boney CM, Quintos JB. Rare complications of pediatric diabetic ketoacidosis. World J Diabetes. 2015;6(1):167-74. doi: 10.4239/wjd.v6.i1.167. [PubMed: 25685287].

14. Felner EI, Klitz W, Ham M, Lazaro AM, Stastny P, Dupont B, et al. Genetic interaction among three genomic regions creates distinct contributions to early- and late-onset type 1 diabetes mellitus. Pediatr Diabetes. 2005;6(4):213-20. doi: 10.1111/j.1399-543X.2005.00132.x. [PubMed: 16390390]. 
15. Oyarzabal Irigoyen $M$, Garcia Cuartero B, Barrio Castellanos R, Torres Lacruz M, Gomez Gila AL, Gonzalez Casado I, et al. Ketoacidosis at onset of type 1 diabetes mellitus in pediatric age in Spain and review of the literature. Pediatr Endocrinol Rev. 2012;9(3):669-71. [PubMed: 22523835].

16. Naeem MA, Al-Alem HA, Al-Dubayee MS, Al-Juraibah FN, Omair A, Al-Ruwaili AS, et al. Characteristics of pediatric diabetic ketoacidosis patients in Saudi Arabia. Saudi Med J. 2015;36(1):20-5. doi: 10.15537/smj.2015.1.9763. [PubMed: 25630000].

17. Jefferies C, Cutfield SW, Derraik JG. 15-year incidence of diabetic ketoacidosis at onset of type 1 diabetes in children from a regional setting. Sci Rep. ;19(5):10358.

18. Wolfsdorf JI, Allgrove J, Craig ME, Edge J, Glaser N, Jain V, et al. ISPAD Clinical Practice Consensus Guidelines 2014. Diabetic ketoacidosis and hyperglycemic hyperosmolar state. Pediatr Diabetes. 2014;15 Suppl 20:154-79. doi: 10.1111/pedi.12165. [PubMed: 25041509].

19. Rewers A, Klingensmith G, Davis C, Petitti DB, Pihoker C, Rodriguez $\mathrm{B}$, et al. Presence of diabetic ketoacidosis at diagnosis of diabetes mellitus in youth: the Search for Diabetes in Youth Study. Pediatrics. 2008;121(5):1258-66. doi:10.1542/peds.2007-1105. [PubMed: 18450868].

20. Dabelea D, Rewers A, Stafford JM, Standiford DA, Lawrence JM, Saydah $\mathrm{S}$, et al. Trends in the prevalence of ketoacidosis at diabetes diagnosis: the SEARCH for diabetes in youth study. Pediatrics. 2014;133(4):938-45. doi: 10.1542/peds.2013-2795. [PubMed: 24685959].

21. Razavi Z, Karimpourian A, Aramian LM, Bazmamoun H. Demographic Characteristics of Type 1 Diabetic Children and Adolescents in Hamadan, Iran. J Res Health Sci. 2015;15(3):196-9. [PubMed: 26411667].

22. Spaans EA, van Dijk PR, Groenier KH, Brand PL, Reeser MH, Bilo HJ, et al. Seasonality of diagnosis of type 1 diabetes mellitus in the Netherlands (Young Dudes-2). J Pediatr Endocrinol Metab. 2016;29(6):657-61. doi:10.1515/jpem-2015-0435. [PubMed: 27008693].

23. Butalia S, Johnson JA, Ghali WA, Southern DA, Rabi DM. Temporal variation of diabetic ketoacidosis and hypoglycemia in adults with type 1 diabetes: A nationwide cohort study. J Diabetes. 2016;8(4):552-8. doi: 10.1111/1753-0407.12336. [PubMed: 26301804]

24. Otieno CF, Kayima JK, Omonge EO, Oyoo GO. Diabetic ketoacidosis: risk factors, mechanisms and management strategies in sub-Saharan Africa: a review. East Afr Med J. 2005;82(12 Suppl):S197-203. [PubMed: 16619733].

25. Razavi Z. Frequency of ketoacidosis in newly diagnosed type 1 diabetic children. Oman Med J. 2010;25(2):114-7. doi:10.5001/omj.2010.31. [PubMed: 22125712].

26. Schober E, Rami B, Waldhoer T, Austrian Diabetes Incidence Study G. Diabetic ketoacidosis at diagnosis in Austrian children in 19892008: a population-based analysis. Diabetologia. 2010;53(6):1057-61. doi: 10.1007/s00125-010-1704-1. [PubMed: 20213235].

27. Guisado-Vasco P, Cano-Megias M, Carrasco-de la Fuente M, Corres-
Gonzalez J, Matei AM, Gonzalez-Albarran O. Clinical features, mortality, hospital admission, and length of stay of a cohort of adult patients with diabetic ketoacidosis attending the emergency room of a tertiary hospital in Spain. Endocrinol Nutr. 2015;62(6):277-84. doi: 10.1016/j.endonu.2015.02.003. [PubMed: 25888157].

28. Syed M, Khawaja FB, Saleem T, Khalid U, Rashid A, Humayun KN Clinical profile and outcomes of paediatric patients with diabetic ketoacidosis at a tertiary care hospital in Pakistan. J Pak Med Assoc. 2011;61(11):1082-7. [PubMed: 22125983].

29. Rodacki M, Pereira JR, Nabuco de Oliveira AM, Barone B, Mac Dowell R, Perricelli $\mathrm{P}$, et al. Ethnicity and young age influence the frequency of diabetic ketoacidosis at the onset of type 1 diabetes. Diabetes Res Clin Pract. 2007;78(2):259-62. doi:10.1016/j.diabres.2007.04.002. [PubMed: 17478002].

30. Blanc N, Lucidarme N, Tubiana-Rufi N. Factors associated with childhood diabetes manifesting as ketoacidosis and its severity. Arch Pediatr. 2003;10(4):320-5

31. Jesic MD, Jesic MM, Stanisavljevic D, Zdravkovic V, Bojic V, Vranjes M et al. Ketoacidosis at presentation of type 1 diabetes mellitus in children: a retrospective 20-year experience from a tertiary care hospital in Serbia. Eur J Pediatr. 2013;172(12):1581-5. doi: 10.1007/s00431-0132083-7. [PubMed: 23835857].

32. Al-Yaarubi S, Ullah I, Sharef SW, Al Shidhani A, Al Hanai S, Al Kalbani $\mathrm{R}$, et al. Demographic and clinical characteristics of type 1 diabetes mellitus in omani children - single center experience. Oman Med J. 2014;29(2):119-22. doi:10.5001/omj.2014.29. [PubMed: 24715939].

33. Bui H, To T, Stein R, Fung K, Daneman D. Is diabetic ketoacidosis at disease onset a result of missed diagnosis?. J Pediatr. 2010;156(3):472-7. doi: 10.1016/j.jpeds.2009.10.001. [PubMed: 19962155].

34. Habib HS. Frequency and clinical characteristics of ketoacidosis at onset of childhood type 1 diabetes mellitus in Northwest Saudi Arabia. Saudi Med J. 2005;26(1):1936-9.

35. Russo A. [Acute abdominal pain in diabetic ketoacidosis, the possible cause of diagnostic error. Review of 3 clinical cases]. Minerva Med. 1987;78(19):1449-51. [PubMed: 3118253].

36. Umpierrez GE, Murphy MB, Kitabchi AE. Diabetic Ketoacidosis and Hyperglycemic Hyperosmolar Syndrome. Diabetes Spectrum. 2002;15(1):28-36. doi: 10.2337/diaspect.15.1.28.

37. Carlotti AP, St George-Hyslop C, Bohn D, Halperin ML. Hypokalemia during treatment of diabetic ketoacidosis: clinical evidence for an aldosterone-like action of insulin. J Pediatr. 2013;163(1):207-12 e1. doi: 10.1016/j.jpeds.2013.01.007. [PubMed: 23410602].

38. Kanwal SK, Bando A, Kumar V. Clinical profile of diabetic ketoacidosis in Indian children. Indian J Pediatr. 2012;79(7):901-4. doi: 10.1007/s12098-011-0634-3. [PubMed: 22207489].

39. Chiarelli F, Marcovecchio ML. DKA management and outcomes. Int Pediatr Endocrinol. 2013;1(16). 